\title{
Ici et ailleurs : les productions vidéographiques nigérianes, cultures et techniques en réseaux
}

\section{Benoît Turquety}

\section{(2) OpenEdition}

\section{Journals}

Édition électronique

URL : http://journals.openedition.org/edl/2501

DOI : 10.4000/edl.2501

ISSN : 2296-5084

Éditeur

Université de Lausanne

\section{Édition imprimée}

Date de publication : 15 décembre 2017

Pagination : 175-194

ISBN : 978-2-940331-66-6

ISSN : 0014-2026

\section{Référence électronique}

Benoît Turquety, «Ici et ailleurs : les productions vidéographiques nigérianes, cultures et techniques en réseaux », Études de lettres [En ligne], 3-4 | 2017, mis en ligne le 15 décembre 2019, consulté le 17 décembre 2020. URL : http://journals.openedition.org/edl/2501; DOI : https://doi.org/10.4000/edl. 2501

\section{(C) Études de lettres}




\section{ICI ET AILLEURS: \\ LES PRODUCTIONS VIDÉOGRAPHIQUES NIGÉRIANES, CULTURES ET TECHNIQUES EN RÉSEAUX}

Cet article s'intéresse aux conditions d'émergence et de diffusion, au début des années 1990, d'une réelle industrie de production de films de fiction au Nigéria marquant une rupture avec la production africaine. En utilisant la vidéo plutôt que la pellicule qui impliquait une forte dépendance vis-à-vis de l'Occident, le centre de production prolifère, mêle différentes cultures et formes et s'émancipe peu à peu.

\section{Un cinéma "véritablement africain"?}

L'ethnologue et cinéaste Jean Rouch, rédigeant pour l'UNESCO en 1961 un rapport intitulé Situation et tendances actuelles du cinéma africain, reprenait dès sa première page une citation de l'historien du cinéma Georges Sadoul :

65 ans après l'invention du cinéma, en 1960, il n’a pas encore été produit, à ma connaissance, un seul long métrage véritablement africain, je veux dire interprété, photographié, écrit, conçu, monté, etc., par des noirs et parlant bien entendu une langue noire. Ainsi 200 millions se sont donc vu interdire la forme la plus évoluée du plus moderne des arts. Je suis persuadé qu’avant la fin des années 1960, ce scandale ne sera plus qu'un mauvais souvenir de temps révolus ${ }^{1}$.

I. G. Sadoul, "Le marché africain", Afrique action (Tunis), 1961 ; cité par J. Rouch, Situation et tendances actuelles du cinéma africain, p. 1 sq. 
Bien sûr, des films avaient été tournés en Afrique, parfois pour un public africain; mais ils relevaient de la production coloniale, films de propagande ou d'éducation, conçus selon les stéréotypes occidentaux, et dirigés par des cadres issus des administrations européennes. Ces films parlaient les langues des colonisateurs, de toutes les manières possibles: d'abord parce qu'on n'y entendait que le français ou l'anglais; ensuite, parce que la forme des films y était adaptée à ce que les colons pensaient que les Africains pourraient voir, comprendre, ou apprécier ${ }^{2}$. Après quelques tentatives au milieu des années 1950, ce sont effectivement les années 1960 qui virent émerger quelque chose comme le cinéma "véritablement africain» décrit par Georges Sadoul - notamment avec Mandabi, tourné en wolof par Sembène Ousmane en 1968, et Cabascabo, réalisé la même année en zarma par Oumarou Ganda. Mais ces deux films sont encore des coproductions françaises. En fait, l'immense majorité du cinéma africain qui suivit ce que certains nommèrent les «indépendances nominales ${ }^{3}$ fut partiellement ou totalement financé par l'Occident, qui fournissait de plus l'infrastructure technique manquante. Un pays aussi important à tous points de vue que le Nigéria par exemple, le plus peuplé d'Afrique et ancienne colonie britannique qui bénéficia d'un boom pétrolier dans les années 1970, ne disposa jamais de laboratoire de développement photochimique. Jusque dans les années 1980, alors que le cinéma ne se produisait que sur pellicule, «[t]oute la post-production était faite à l'étranger, le plus souvent à Londres, car il n'y avait pas d'installations au Nigéria, et les coûts de post-production (surtout le développement de la pellicule) écrasaient rapidement toutes les autres lignes budgétaires du film " ${ }^{4}$. Même donc lorsque le cinéma africain a commencé d'exister réellement, il n’a jamais véritablement acquis une complète indépendance. Il est resté rattaché aux réseaux techniques et économiques occidentaux. Cela a impliqué en retour une autre dépendance, vis-à-vis des circuits occidentaux de légitimation culturelle et artistique.

Car bien sûr, parallèlement, les Africains voyaient des films. Les salles de cinéma n'ont jamais été très nombreuses, en proportion de la densité

2. Sur ces aspects, voir L. Grieveson, C. MacCabe (eds), Empire and film.

3. Voir par exemple F. Kodjo, «Les cinéastes africains face à l'avenir du cinéma en Afrique», p. 607.

4. J. L. Miller, Nollywood Central, p. 12. 
européenne, et elles n’ont jamais pénétré le milieu rural. Mais les grandes métropoles africaines ont toutes vu s'en construire quelques-unes, et selon les pays, les campagnes pouvaient également voir circuler des unités mobiles de projection, dont les structures se sont parfois maintenues après la période coloniale. Ainsi que le résumait Brian Larkin pour le cas nigérian:

Spécialistes et historiens du cinéma ont eu tendance à traiter le cinéma ambulant comme s'il était borné historiquement, commençant dans les années 1930 et se terminant autour de la période de l'indépendance [...]. Après l'indépendance, les unités de cinéma mobile ont été maintenues par l'État indépendant et connurent même une importante expansion. Toujours largement centrés sur le film éducatif, promouvant l'État (maintenant postcolonial), les majigi du nord du Nigéria se maintinrent jusqu'à l'émergence de la télévision ${ }^{5}$.

Ces unités mobiles ont donc diffusé le cinéma un peu partout; elles sont toutefois restées en dehors des circuits commerciaux: elles ne montraient pas de films de fiction et de divertissement, et les séances n'étaient pas payantes. Les salles urbaines commerciales, quant à elles, ont développé une politique de programmation pragmatique fondée sur l'optimisation de la rentabilité : à part quelques cas exceptionnels - notamment l'Égypte, qui a connu une réelle industrie du cinéma florissante et circulant aussi bien à l'étranger que dans le pays même -, les distributeurs africains ne montraient que des films provenant des grands centres mondiaux de production - principalement Hollywood, Mumbai, voire Hong-Kong et quelques autres -, dont les copies après l'exploitation dans les pays occidentaux étaient bradées pour une seconde vie dans les pays du Sud. Les locations se trouvaient ainsi très bon marché, et l'aura culturelle de ces films leur assurait la rentabilité. Mais donc, les Africains ne voyaient pas de films africains - et l'absence de réseau de distribution local rendait à peu près vain l'espoir de construire un secteur cinématographique africain qui pût à terme devenir économiquement viable et indépendant. Dépendant des investissements occidentaux, les films produits en Afrique circulaient plutôt dans les festivals européens et américains, trouvant là la seule possibilité de légitimation qui permettrait aux

5. B. Larkin, Signal and noise, p. 119. 
cinéastes de poursuivre leur œuvre, et à leurs pays d'origine d'acquérir une visibilité sur le plan international.

Le cinéma africain est donc le lieu d'une profonde disjonction. S’il y a de nombreuses manières de devoir considérer qu'il y a non pas un, mais des cinémas africains - comme d'un autre point de vue, l'importance de la problématique panafricaine dans sa construction pourrait justifier le singulier - , la première consiste à distinguer radicalement le cinéma fait par les Africains, du cinéma vu par les Africains. Dans ce domaine, les études sur la production et celles sur la réception sont complètement indépendantes: leurs objets sont restés hermétiquement disjoints, ainsi que leurs enjeux esthétiques, culturels et sociotechniques. Le cinéma de production africaine, depuis les années 1960, s'est pensé et a été massivement reçu dans le cadre anti-impérialiste, anticolonial, des constructions et revendications culturelles nationales ou panafricaines. Ainsi que l'écrivit Jonathan Haynes:

[L]e paradigme qui a gouverné les études sur le cinéma africain [...] a été formé - comme le cinéma africain l'a été lui-même - par le moment historique de la décolonisation et ses idéologies jumelles du nationalisme culturel et d'une révolution sociale plus ou moins de gauche ${ }^{6}$.

Mais la réception quant à elle fut l'un des lieux essentiels de la diffusion continuée des modèles culturels du Nord.

\section{Une rupture: Nollywood, réseaux culturels}

Or ces dernières années, cette situation s'est transformée. Dans le premier tiers des années 1990 a en effet émergé une réelle industrie de production de films de fiction en plein cœur de l'Afrique subsaharienne, à Lagos, Nigéria. Une industrie non seulement viable et autonome, mais dont le développement fut massif, et dont le rayonnement dépassa vite le strict cadre local, linguistique, ou national, pour gagner toute l'A frique, ainsi que le continent sud-américain, et épouser la circulation des diasporas africaines partout dans le monde. Ceci eut lieu sans aucun soutien financier, structurel, technique ou culturel du Nord, ni d'ailleurs des

6. J. Haynes, «Introduction», p. 7. 
instances politiques locales. C'est ainsi que le sondage de l'UNESCO de 2006 a reconnu le Nigéria second producteur de longs métrages de fiction au monde avec 872 films réalisés sur l'année, soit un peu moins que l'Inde - «Bollywood", l'industrie basée à Mumbai, ayant produit 1091 films cette année-là -, et presque deux fois plus que les États-Unis, qui produisirent 485 longs métrages ${ }^{7}$. Comment un tel renversement est-il possible?

Si le phénomène nigérian se révèle toujours plus difficile à circonscrire et à documenter, les histoires s'accordent étonnamment sur son point de départ. En 1992, Kenneth Nnebue, revendeur spécialisé en équipement électronique d'occasion et en cassettes VHS vierges, produisit un film pour quelques centaines de dollars, qu'il fit réaliser et distribua directement en vidéo, Living in Bondage. Il connut un succès phénoménal et immédiat: selon Olivier Barlet, "les chiffres varient de 200'000 à 750 '000 cassettes vendues ${ }^{8}$; et ainsi que le résume Jonathan Haynes:

Living in Bondage 1 a fait immédiatement sensation lorsqu'il est sorti à la fin de 1992, sa réputation se répandant comme une traînée de poudre et uniquement par le bouche à oreille - il n'y avait ni publicité, ni annonces, ni sortie en grande pompe 9 .

Immédiatement, le succès fit de nombreux émules. Toujours selon Barlet:

Dès 1994, la commission de censure recensait 177 films. Les vendeurs de magnétoscopes du quartier Idumota du marché central de Lagos ayant le matériel nécessaire pour les dupliquer, ils ont fait fortune en les distribuant ${ }^{10}$.

En cette année 1994, un autre pas fut franchi: Nnebue produisit Glamour Girls, premier film vidéo nigérian en langue anglaise. Cette décision ouvrit la circulation des films à une tout autre échelle que les productions précédentes, tournées en igbo.

7. «Nigeria surpasses Hollywood as world's second largest film producer», UN News Centre, 5 mai 2009, <http://www.un.org/apps/news/story.asp?NewsID=30707>.

8. O. Barlet, Les cinémas d'Afrique des années 2000, p. 359.

9. J. Haynes, Nollywood, p. 28.

Io. O. Barlet, Les cinémas d'Afrique des années 2000, p. 359. 
L'industrie connut donc une croissance massive. Les chiffres sont difficiles à établir avec certitude, mais comme le montre le sondage de l'UNESCO de 2006, ils sont en tout état de cause phénoménaux. Selon Pierre Barrot en 2005, "[o]n estime que la "video industry" emploie au total entre 250 '000 et 300’000 personnes [au Nigéria], la plupart intervenant dans la commercialisation des vidéos et non dans leur production " ${ }^{11}$. Olivier Barlet rapportait en 2012 que «le nombre de longs métrages de fiction enregistrés au bureau de censure a dépassé le cap du millier en 2004 pour atteindre 1711 en 2006 et 1770 en 2008 (on compte au maximum une trentaine de documentaires par an), sachant que de nombreux films ne lui sont pas présentés", l'ensemble formant une «industrie qui affirme valoir plus de 600 millions de dollars en $2010{ }^{12}$. Elle l'affirme, mais s'il est difficile de le vérifier exactement, c'est parce que ses formidables réseaux, qui permettent la fabrication et la distribution de ces films depuis Lagos jusque partout dans le monde, fonctionnent presque intégralement en dehors de l'économie formelle ${ }^{13}$ - ce qui a pu empêcher, au moins jusqu'à la parution en 2009 du sondage de l'UNESCO, la perception de son existence même.

Le succès est en tout cas incontestable, et peut se mesurer à deux niveaux. Au plan économique d'abord, l'industrie vidéographique de Lagos a fait modèle, et l'Afrique subsaharienne surtout a vu se développer des tentatives similaires. À Kano, dans ce nord du Nigéria de culture musulmane et soumis à la charia, une production vidéo s'est construite sur des bases un peu différentes, plus proches des comédies musicales produites à Mumbai, mieux adaptées aux critères moraux de la culture haoussa. À Accra, dans le Ghana voisin, peu après l'émergence $\mathrm{du}$ "Nollywood» nigérian est née "Ghollywood» ${ }^{14}$. Plus tard, Dar es Salaam, en Tanzanie, a vu naître "Bongowood", et Nairobi, Kenya, "Riverwood ${ }^{15}$, deux des plus importantes dérivations de l'original - mais le Burkina Faso, l'Ouganda, la République démocratique du

II. P. Barrot (éd.), Nollywood, p. 45.

I2. O. Barlet, Les cinémas d'Afrique des années 2000, p. 361 et 363.

13. R. Lobato, «Nollywood at large».

I4. Sur le cas ghanéen, voir notamment B. Meyer, «Ghanaian popular video movies between state film policies and Nollywood".

I5. Décrite par O. Barlet comme "production spontanée de vidéos tournées dans les langues locales dont les marchands se trouvent regroupés à River Road à Nairobi» (Les cinémas d'Afrique des années 2000, p. 366). 
Congo, l'Éthiopie, en connaissent tous des versions de plus ou moins grande envergure ${ }^{16}$. Cette exportation du modèle nigérian s'opère sur l'ensemble de la structure techno-économique: méthodes et moyens de tournage, réseaux de distribution, etc. Certaines thématiques, certains choix formels circulent également, mais se trouvent chaque fois réadaptés aux cultures et enjeux locaux.

Cette dimension culturelle constitue le second niveau auquel se mesure le poids de Nollywood. Statistiques et témoignages sont sans appel: la production vidéographique nigériane a influencé massivement non seulement les fictions produites dans le pays et sur le continent, mais également les cultures de manière plus générale. Ainsi, Ibbo Daddy Abdoulaye racontait qu'au Niger:

Les moindres faits et gestes des vedettes de ces films populaires sont souvent plus connus que certaines questions d'intérêt national, notamment grâce aux posters qui tapissent les murs et surtout aux magazines en langue haoussa comme Fina Fina ou Dunyar Fim, au point que des esprits malins, flairant le filon, se sont lancés dans l'imitation de ces derniers et miment leurs paroles et leurs actes lors de certaines cérémonies comme baptêmes et mariages contre monnaie sonnante et trébuchante ${ }^{17}$.

Tunde Oladunjoye rapportait quant à lui en 2005 des propos de Don Pedro Obaseki :

Les films sont, aujourd'hui, le meilleur moyen de promotion du Nigéria. J'étais au Libéria il y a deux ans pour une interview avec certains chefs de la rébellion. On m'a emmené au Nord du pays, dans un coin perdu. En plein cœur de la brousse, ils ont sorti un magnétoscope et un téléviseur et - incroyable mais vrai! - les rebelles se passaient des films nigérians ${ }^{18}$.

Ailleurs encore, la présence de ces films est remarquée pour ses répercussions notables dans l'ensemble de la culture nationale, ainsi que le note Ogova Ondego :

I6. J. L. Miller, Nollywood Central, p. 86, et travail de master en cours de Sarah Imsand, UNIL.

I7. I. D. Abdoulaye, "Niger: les films nigérians au "banc des amoureux" ", p. 103.

I8. T. Oladunjoye, "Un train en marche», dans P. Barrot (éd.), Nollywood, p. 72. 
Les Kenyans s'inspirent également de la mode vestimentaire nigériane. [...] Certaines personnes adoptent les noms de personnages - «Awilo Sharp Sharp" et "Aki na Ukwa» - vus dans des films nigérians. Ces films cultivent la fierté d'être Africain chez les spectateurs kenyans. Ils valorisent le fait de s'habiller à l'africaine et défendent certains critères de beauté locaux, en particulier le goût pour les grosses femmes ${ }^{19}$.

Enfin, cette pénétration dépasse les limites géographiques du continent, puisque jusqu’à Sainte-Lucie (Antilles), «les films vidéo nigérians et ceux de l'industrie ghanéenne voisine sont de loin les DVD piratés les plus vendus dans ces boutiques de trottoir d'une ville où presque tous les médias sont des copies illégales de leurs originaux» ${ }^{20}$.

Ces témoignages, dans leur diversité, donnent la mesure d'un phénomène; mais il s'agit d'en souligner également la nouveauté assez profonde, qui explique également son ampleur. Ainsi que le résumait le producteur Teco Benson: "Pour la première fois, nos films ne sont pas tournés dans une perspective européenne mais disent la vérité africaine» ${ }^{21}$. Si leur rapport avec une "vérité africaine» est complexe et discuté $^{22}$, il n'en reste pas moins la justesse et l'importance du constat de cette "première fois». Pour la première fois, des films sont produits sur le continent africain, par des équipes de production et de réalisation entièrement africaines, sans aucune intervention financière ou artistique provenant du Nord. De manière plus cruciale encore peut-être, ces films ont été pensés, fabriqués et diffusés massivement sans jamais passer par les circuits de légitimation culturels occidentaux (festivals, distribution en salles en Europe ou aux États-Unis). C'est la première fois. Nollywood pourrait bien représenter la véritable matérialisation inaugurale de ce "cinéma véritablement africain" que Sadoul appelait de ses vœux - même si bien sûr, cet énoncé, qui renvoie à une industrie dans son ensemble, pourrait être perçu comme une négation du long, acharné et somptueux travail des cinéastes qui ont réfléchi formellement et institutionnellement aux moyens de donner vie à un cinéma authentiquement africain, et qui y ont largement réussi, même sans toujours atteindre à

19. O. Ondego, «Le Kenya sous dépendance», dans P. Barrot (éd.), Nollywood, p. 120.

20. O. Okome, «Nollywood and its critics», p. 30.

2I. Propos rapportés par O. Barlet, Les cinémas d'Afrique des années 2000, p. 363.

22. Voir notamment O. Okome, "Nollywood and its critics». 
l'autonomie complète dont ils rêvaient. C'est à eux d'abord qu'est incombée la tâche de lutter contre les schémas occidentaux sans pouvoir les ignorer complètement, mais aussi de se demander en quoi pouvait concrètement consister une esthétique cinématographique africaine.

On peut bien sûr se demander d'ailleurs ce que signifie exactement ce "véritablement africain". Cette question se pose de manière générale - pour reprendre l'argument bien connu: de qui dirait-on ici qu'il fait un cinéma "véritablement européen"? -, mais plus encore peut-être dans le cas de Nollywood, dont les films ne renvoient qu'exceptionnellement aux thématiques de l'africanité, du (post-)colonialisme, etc. De fait, ces œuvres trouvent leurs origines à la fois dans des formes africaines, locales - de plus ou moins longue lignée - et dans des ensembles esthétiques transnationaux. L'une des sources les plus importantes, dont la filiation peut apparaître à maints égards comme la plus directe, est le théâtre itinérant populaire yoruba. Cette tradition, établie depuis les années 1940 sur la base d'une synthèse de diverses formes plus anciennes, mobilise religion, musique, arts oratoires, etc., et connut un succès grandissant. Dans les années 1960-1970, une centaine de troupes sillonnait toute la zone linguistique yoruba ${ }^{23}$. Des films furent réalisés en pellicule, puis on enregistra des performances en vidéo, qui étaient projetées dans les villages accompagnées des acteurs de la troupe. Mais Nollywood s'alimente également massivement à des sources culturelles extérieures, formes circulant par de vastes réseaux mondiaux de production massive et de réappropriations, créolisations, reprises, copies et réinventions. Il s'agit notamment du mélodrame, dans sa version hollywoodienne classique, ainsi que de ses dérivations en soap operas, qui peuvent être étasuniennes, mais aussi - voire surtout - latino-américaines. La télévision nigériane - comme nombre de ses consœurs africaines - montrait effectivement abondamment ces feuilletons télévisuels mexicains ou brésiliens littéralement interminables ${ }^{24}$ - à l'image du célébrissime Los ricos también lloran importé du Mexique. L'impact de ces formes est massif partout dans le monde, et Nollywood y a puisé certains aspects de ses intrigues, de ses principes de montage, de son goût pour la stylisation du jeu des acteurs - qui vient aussi du théâtre yoruba -, et de son attirance pour les formats

23. K. Barber, The generation of plays.

24. Voir notamment I. D. Abdoulaye, "Niger: les films nigérians au "banc des amoureux"", p. 72 et 103. 
longs - sans commune mesure avec un soap opera, les films nollywoodiens tendent tout de même à se jouer en deux parties de trois heures, loin de la norme étasunienne traditionnelle des nonante minutes.

Malgré cette hybridité profonde travaillée par des réseaux culturels interagissant à des échelles radicalement différentes, il y a tout de même dans ces films une dimension africaine pleinement perçue comme telle, qui renvoie à certaines spécificités, goûts, valeurs, problèmes - elle s'entend notamment aux remarques d'Ogova Ondego sur leur influence au Kenya. Le succès de Nollywood, en lui-même autant que par les œuvres produites, participe d'une valorisation de certaines formes de la culture africaine contemporaine, qui ne sont pas rattachées aux arts ethniques ou traditionnels. Elles relèvent plutôt de ce que l'on pourrait appeler avec Karin Barber les "arts populaires» 25 - dont Haynes a pu faire l'hypothèse qu'ils constituaient le meilleur modèle pour comprendre les formes et enjeux de la production nollywoodienne ${ }^{26}$.

Ce succès - financier et culturel - n'implique pourtant pas une simple légitimation de ces formes. Nollywood est resté un objet problématique, au Nigéria comme ailleurs. Onookome Okome a présenté en 2010 les motifs principaux de la réticence de l'intelligentsia nigériane à l'égard de la production vidéographique nationale ${ }^{27}$, qui repose sur des réserves de plusieurs types. Le premier problème concerne l'image du Nigéria telle qu'elle est construite par Nollywood et diffusée à l'étranger: la sorcellerie notamment tient une place de choix dans les intrigues, l'appétit de richesse y apparaît parfois comme l'unique motivation, etc. Ogova Ondego écrivait ainsi :

On attribue aux vidéos nigérianes plusieurs conséquences sur le Kenya. On dit que le thème de la sorcellerie influence les Kenyans dans le sens $\mathrm{du}$ « retour en arrière» et de l'«immoralité ${ }^{28}$.

Cet effet, pour l'élite cultivée, s'accentue d'un constat de faiblesse technique et esthétique des films, qui sont réalisés en très peu de temps et avec un budget souvent dérisoire, et accusés d'être eux-mêmes conçus

25. K. Barber, «Popular arts in Africa».

26. Voir notamment J. Haynes, Nollywood, p. 5.

27. O. Okome, "Nollywood and its critics».

28. O. Ondego, "Le Kenya sous dépendance», dans P. Barrot (éd.), Nollywood, p. 119. 
dans l'unique but d'enrichir leurs fabricants. Il s'agit bien sûr de maintenir les questions techniques et esthétiques sur deux plans nettement distincts; mais en l'occurrence, l'intrication des deux est liée aux circonstances précises qui caractérisent depuis ses commencements le phénomène nollywoodien.

\section{La vidéo: nouveaux réseaux techniques}

C'est donc au début des années 1990 qu'a émergé cette industrie. Le Nigéria n'avait alors jamais réussi à construire quelque chose comme une cinématographie, même à l'échelle restreinte qui fut celle d'autres pays du continent; mais la mise en place au milieu des années 1980 du Structural Adjustment Program - qui impliqua notamment une dévaluation spectaculaire du naira - acheva les velléités de réalisation cinématographique qui avaient pu subsister chez quelques acharnés:

Tout ce qui impliquait une monnaie forte se trouvait soudain hors de portée du budget de n'importe quel cinéaste, notamment l'importation de pellicule et le voyage à Londres pour le traitement des images et la post-production, rien de tout cela ne pouvant se faire au Nigéria ${ }^{29}$.

Jusqu'alors en effet, le tournage d'une fiction s'envisageait sur pellicule, 35 ou $16 \mathrm{~mm}$, ce qui implique d'abord des équipes plus ou moins nombreuses et expertes, mais aussi un réseau ou un ensemble technique complexe ${ }^{30}$. Les machines qui structurent ce réseau, caméra et enregistreur notamment, coûtent passablement cher, sans doute; mais pour le Nigéria - comme pour l'ensemble des pays africains - cet aspect était relativement plus facile à gérer que l'approvisionnement en pellicule et surtout les opérations chimiques de développement du négatif et tirage des copies positives, opérations qui nécessitent des laboratoires dont le pays ne dispose pas, et appellent donc le transport des bobines de film jusqu'en Europe. Tout cela générait des surcoûts en livres sterling, outre une importante complexité organisationnelle. Parallèlement, la situation politique difficile du Nigéria engendrait en même temps une très grande

29. J. Haynes, Nollywood, p. 6.

30. J'entends ces notions au sens de Gilbert Simondon (Sur la technique). 
insécurité, qui entraîna la fermeture de la quasi-intégralité des salles de cinéma...

C'est dans cette situation de blocage qu'intervint la décision prise par Kenneth Nnebue et ses collaborateurs, notamment Okechukwu Ogunjiofor, de tourner un film de fiction non pas sur pellicule argentique, mais en vidéo - plus précisément en Super VHS ou S-VHS, format vidéo introduit en 1987 par la compagnie japonaise Victor. L'enregistrement des images et des sons sur bande magnétique était extraordinairement moins coûteux que la réalisation de films sur pellicule argentique, et offrait en outre une relative autonomie technique. Aucun laboratoire, aucun traitement chimique complexe n'était plus nécessaire; le montage s'opérait à l'aide d'un magnétoscope supplémentaire, tout à fait abordable. Il était donc aisé de produire ensuite des copies sur cassettes VHS de l'original, lisibles par n'importe quel magnétoscope de salon. Ceux-ci étaient déjà notablement présents au Nigéria parmi les classes moyennes; en retour, le succès des films garantirait une diffusion de beaucoup plus grande ampleur de ces lecteursenregistreurs ${ }^{31}$. L'arrivée des images directement dans l'espace privé permettait d'éviter les salles de cinéma, dangereuses et de mauvaise réputation. Des conditions collectives de réception se reconstruisirent à partir de ce contexte familial de visionnement, pour inclure autour de téléviseurs des voisinages plus ou moins distants, dans des "video parlors» de plus ou moins grande capacité ${ }^{32}$.

Le basculement de la pellicule argentique à la vidéo ne permettait donc pas seulement une réduction massive des coûts de fabrication, qui seule rendait possible la réalisation de films dans le contexte économique $\mathrm{du}$ lieu et du moment. Il engendrait également une transformation radicale du réseau technique de production, et du mode de circulation des films dans la société. Du côté de la réalisation, c'est d'abord un changement d'échelle du réseau qui fut crucial: au lieu de devoir, avec la pellicule, mobiliser de manière permanente un circuit transnational complexe de déplacement d'objets, de compétences et d'argent, la vidéo permettait - une fois l'achat initial du matériel effectué, pour lequel Nnebue a dû se déplacer exprès au Japon ${ }^{33}$ - de fonctionner de manière

3I. J. Haynes, Nollywood, p. 11.

32. Cf. M. Şaul, R. A. Austen (eds), Viewing African cinema in the twenty-first century.

33. J. Haynes, Nollywood, p. 31. 
quasi autonome sur la base d'un réseau technique minimal disponible au niveau le plus local. Tournage, montage, duplication des cassettes VHS ou plus tard des VCD ${ }^{34}$, l'ensemble des opérations depuis l'idée initiale jusqu'à la mise à disposition des copies finales et au visionnement peuvent être réalisés dans un rayon très restreint, dans la périphérie de Lagos, de Kano ou d'Accra, ne demandant qu'une infrastructure légère. Le seul indispensable prérequis est le courant électrique, ce qui au Nigéria n'est pas une évidence ${ }^{35}$; il s'agit donc de disposer de groupes électrogènes pour pallier toute coupure malvenue. D'autres problèmes peuvent bien sûr se poser; notamment, le matériel vidéo est plus fragile que le matériel de tournage sur pellicule, moins bien adapté au climat africain, et demande pour la maintenance et la réparation des compétences plus spécifiques a priori - même si des bricolages ponctuels sont parfois possibles. En outre, l'Afrique n'est pas intégrée - à cette époque en tout cas - aux réseaux de distribution de pièces de rechange, ce qui peut rendre une machine complètement inutilisable pendant une assez longue durée. Faute de pouvoir peser sur la forme de ces réseaux industriels transnationaux hiérarchisés selon la priorité des marchés, il s'agit donc de se maintenir dans une gamme d'équipement aussi peu onéreux que possible, de manière à pouvoir au besoin simplement remplacer une machine défectueuse.

La diffusion des copies aurait pu être compliquée à réaliser, puisqu'il s'agissait de distribuer sur une zone géographique aussi large que possible des objets physiques, cassettes ou disques. Mais Brian Larkin ou Ramon Lobato ont montré que le succès immédiat de Living in Bondage et de la production vidéo des années suivantes a été possible parce que les cassettes VHS ont emprunté les circuits de commerce informel qui étaient déjà en place et avaient éprouvé et démontré leur efficacité - y circulaient d'autres productions médiatiques, par exemple les cassettes audio portant des enregistrements souvent piratés de musique locale ou occidentale, discours ou sermons, les cassettes VHS portant des copies

34. Le VCD (Video CD) est la forme dominante de support vidéo numérique en Asie, en Afrique, et dans l'ensemble des pays non occidentaux, où le DVD n'occupe qu'une place restreinte. Présentant une image de 352 x 288 pixels (au format PAL), sa résolution équivaut environ au quart d'une image télévisée classique. Il a l'avantage du faible coût, et ne permet pas le cryptage des images ou la protection contre la copie.

35. C'est sur les légendaires problèmes électriques du pays que J. Haynes ouvre Nollywood, p. Xv. 
illégales de films occidentaux, etc. Ces circuits étaient entretenus par les vendeurs de matériel électronique, tel Kenneth Nnebue, afin de soutenir le commerce des lecteurs de ces médias. Si Nollywood a ensuite régulièrement tenté de lutter contre le piratage, son incapacité à éradiquer le phénomène est liée au fait qu'elle repose profondément sur une «infrastructure de piraterie» ${ }^{36}$. Ainsi que l'a écrit Brian Larkin:

L'industrie nouvelle a inauguré de nouveaux genres cinématographiques et engendré un mode de reproduction et de distribution entièrement original, qui utilise les capitaux, l'équipement, le personnel et les réseaux de distribution des médias pirates. Ces vidéos nigérianes sont une forme médiatique légale qui ne pourrait pas exister sans l'infrastructure créée par son double illégal, les médias pirates ${ }^{37}$.

Cette infrastructure préexistant et s'auto-engendrant à mesure de son expansion, les copies pirates des vidéos réalisées par les vendeurs à la sauvette minant et à la fois soutenant le réseau, la distribution put s'étendre géographiquement à partir de pôles restreints jusqu'à couvrir et dépasser le continent, sans qu'aucun investissement spécifique fût nécessaire.

Le passage de la pellicule argentique à la bande magnétique a ainsi provoqué une complète refonte des réseaux sociotechniques de fabrication et de circulation des productions médiatiques, ainsi que des modes de leur inscription dans le milieu social. Ces réseaux organisent chaque fois une géographie spécifique, mettant en jeu des hiérarchies entre pôles et périphéries, pondérées de coefficients plus ou moins importants de centralisation. La production est ainsi passée d'un système argentique transnational à géographie très vaste, à un système magnétique hyper localisé, non seulement au niveau national, mais même au niveau du centre urbain. La diffusion quant à elle a basculé d'un réseau formel à organisation centralisée, focalisé sur un nombre limité de points importants à la répartition calculée sur le territoire - les salles de cinéma - à un réseau informel décentralisé, initié dans le centre urbain de production (Lagos par exemple), mais démultiplié par des interventions locales disséminées, dont ni le nombre ni l'activité ne peuvent être entièrement contrôlés.

36. B. Larkin, "Degraded images, distorted sounds».

37. Ibid., p. 290. 
Cet agencement inédit est ce qui a permis la réalisation des vidéos nollywoodiennes, l'émergence d'une industrie de production relativement stable et prospère, et l'impact culturel massif de ces œuvres. Il s'est aussi constitué en modèle éventuellement exportable ${ }^{38}$, voire transférable sur d'autres secteurs d'activités, exemple d'une réussite locale totalement dénuée de lien concret ou symbolique aux anciennes puissances coloniales, fondée uniquement sur les pratiques et fonctionnements africains.

\section{La vidéo: enjeux esthétiques}

Du point de vue qui nous occupe toutefois, ce réseau de réseaux techniques, sociaux et culturels, ce système complexe et extrêmement spécifique de production - et de diffusion - se matérialise du point de vue esthétique dans les œuvres mêmes.

C'est peut-être avec la vidéo analogique des années 1990 que cette dimension est la plus frappante. Comme je l'ai signalé déjà, ces films étaient tournés sur le matériel vidéo le moins onéreux possible, avec un format - le VHS ou le S-VHS, un petit peu "supérieur» ${ }^{39}$ - destiné à l'amateur grand public, le plus basique de tous les formats vidéo de l'époque, destiné à l'enregistrement d'émissions de télévision et au film de famille, un format quaucun professionnel européen n'aurait accepté d'utiliser dans le cadre de ses activités - sinon éventuellement pour garder un souvenir des vacances familiales ${ }^{40}$. Ce format produisait des images d'une assez faible résolution, et disposant d'une palette de couleurs limitée - ce qui dans un contexte africain est problématique. D'autre part, dans les systèmes analogiques, la duplication entraîne toujours une dégradation: des distorsions apparaissent dans la bande sonore, et des artefacts dans l'image: lignes manquantes, pertes de synchronisation du flux, diminution de netteté des contours... L'importance de la piraterie dans le système de distribution implique que l'on peut avoir affaire à une copie non pas issue directement de l'original, mais de deuxième,

38. O. Barlet, "Le modèle nigérian de la vidéo domestique est-il exportable?».

39. Cet adjectif ne renvoie pas ici à une "qualité» visuelle de l'image, mais à la capacité technique de stockage d'information. Ces deux aspects ne doivent bien sûr pas être confondus. Je me permets de renvoyer ici à B. Turquety, Inventer le cinéma, p. 16.

40. Le VHS est par ailleurs alors le format de lecture le plus largement répandu partout dans le monde, dominant massivement le marché de la vidéo domestique. 
troisième, quatrième génération... En outre, dans ce type d'organisation, l'opération de sous-titrage nécessite dès l'origine une génération supplémentaire de reproduction de l'image, et donc une dégradation. Or bien sûr, la multiplicité des dialectes même à l'intérieur du Nigéria, voire des accents au sein de l'anglais, donne au sous-titrage une place considérable: Living in Bondage était en igbo, langue devenue minoritaire dans la production nollywoodienne, au profit du yoruba et de l'anglais - mais un anglais requérant parfois le sous-titrage pour la circulation internationale. Ceci étant dit, les solutions à la tension entre le caractère local de la production nigériane et la circulation transnationale des films ne sont pas toujours techniques; elles passent parfois par une réappropriation locale des œuvres. Comme le décrit Ogova Ondego :

Les personnages nigérians ont beau parler un anglais difficile à saisir, ce n'est pas un obstacle pour beaucoup de Kenyans, qui se chargent de traduire les dialogues en swahili, en kikuyu ou en anglais, pendant les diffusions dans les vidéo-clubs ou des salles de fortune. Ces commentateurs expliquent ce qui se passe, au fur et à mesure de la projection ${ }^{41}$.

Le rendu déjà très singulier de la VHS est donc transformé encore par la multiplication des copies, pour donner finalement à l'ensemble de la production nollywoodienne de ces années analogiques une cohérence visuelle spécifique, celle des «images dégradées et sons déformés", jeu du signal et du bruit qui fonde, pour l'anthropologue Brian Larkin, ce qui s'élabore finalement en une esthétique propre ${ }^{42}$.

Mais tourner en vidéo a d'autres implications encore. Filmer en Afrique pose des problèmes particuliers pour plusieurs raisons, mais notamment à cause du fait que le matériel - d'origine européenne, étasunienne ou japonaise - n'est pas pensé pour être vendu dans ces pays, et donc pas conçu pour être utilisé dans les conditions qui leur sont propres. Par exemple, la VHS ne dispose que d'une latitude d'exposition extrêmement réduite. Il lui est impossible de reproduire correctement, dans la même image, une zone très claire et une zone très sombre: soit l'une sera "brûlée» - uniformément blanche, sans aucun détail visible -, soit l'autre sera «bouchée» - uniformément noire - soit les deux à la

4I. O. Ondego, «Le Kenya sous dépendance», dans P. Barrot (éd.), Nollywood, p. 119.

42. Voir B. Larkin, "Degraded images, distorted sounds" et Signal and noise. 
fois si l'écart est un peu important. Excessivement surexposée, la zone blanche pourra même irradier le reste de l'image, les couleurs seront faussées, etc. Cette réaction est bien sûr difficile à rendre compatible avec l'éclatante lumière africaine, les surfaces claires des sols et murs illuminés faisant un contraste beaucoup trop fort avec les ombres profondes. Il est par exemple impensable, dans ces conditions, de tourner une scène d'intérieur avec une fenêtre ouverte: on ne pourrait pas intégrer dans une même image vidéo de tels contrastes. C'est une contrainte majeure. Les intérieurs de Living in Bondage sont ainsi tous aveugles, les fenêtres absentes ou bouchées de rideaux, donnant au film dans son ensemble une tonalité nocturne presque étrange, accentuant la sensation de fermeture qui cerne le personnage principal.

Le passage à la vidéo numérique a un peu changé la donne. Ces caméras récentes, même destinées aux amateurs, ont une latitude limitée certes, mais plus importante tout de même que les VHS des années 1990. On peut tourner en extérieurs, et en intérieurs en lumière naturelle - avec certaines précautions. Il reste difficile de jouer avec les ombres de manière aussi souple qu'avec les caméras professionnelles haut de gamme du cinéma occidental, ou avec de la pellicule. Reste aussi que les budgets et les pratiques nollywoodiennes continuent majoritairement d'exclure l'étalonnage et le mixage, ces opérations techniques d'homogénéisation sur l'ensemble d'un film des rendus visuel et sonore. Les films laissent donc entendre, d'une séquence à l'autre, de grandes variations dans la présence et le timbrage des voix, ainsi que dans le rendu des tons des peaux, des contrastes et des palettes colorées. Cela aussi participe de leur esthétique propre - une chance donnée à la reprise de la critique de l'idéologie occidentale de la continuité, entamée par la modernité depuis Pound ou Brecht et reprise ici peut-être, quoique sur d'autres bases et avec d'autres moyens.

Nollywood a donc provoqué, dans le paysage mondial des images animées, une profonde reconfiguration des réseaux culturels et techniques. Son importance tient à n'avoir pas seulement réorienté la circulation des objets entre des pôles préexistants; mais au-delà, à transformer radicalement ces réseaux par un double jeu d'une part de massifs changements d'échelle, et d'autre part de redéfinition des centres et des périphéries. Là où la production en pellicule reposait sur de vastes réseaux transnationaux techniques et financiers impliquant une forte dépendance vis-à- 
vis des anciennes colonies, la vidéo s’appuie sur un réseau local restreint à une zone urbaine: Lagos était la périphérie de Londres, elle devient un centre quasi autonome. Là où la diffusion en pellicule était géographiquement ponctuelle, centralisée, exclusive, elle se fait proliférante, incontrôlée, et tout en dehors des instances de légitimation. Elle mêle trop de cultures et de formes pour ne revendiquer aucun ancrage strictement local; elle n'affirme de fait qu'une chose nouvelle peut-être: n'avoir aucun besoin de l'Occident.

Benoît Turquety

Université de Lausanne 


\section{BIBLIOGRAPHIE}

Abdoulaye, Ibbo Daddy, "Niger: les films nigérians au "banc des amoureux" ", in Nollywood. Le phénomène vidéo au Nigéria, éd. par Pierre Barrot, Paris, L'Harmattan, 2005, p. 101-108.

Barber, Karin, "Popular arts in Africa», African Studies Review, 30/3 (1987), p. 1-78.

-, The generation of plays: Yoruba popular life in theater, Bloomington, Indiana University Press, 2000.

BARlet, Olivier, "Le modèle nigérian de la vidéo domestique est-il exportable?", Africultures, 2003, en ligne: <http://africultures. com/le-modele-nigerian-de-la-video-domestique-est-ilexportable-2764/>.

—, Les cinémas d'Afrique des années 2000. Perspectives critiques, Paris, L'Harmattan, 2012 (coll. Images plurielles).

Barrot, Pierre (éd.), Nollywood. Le phénomène vidéo au Nigéria, Paris, L'Harmattan, 2005 (coll. Images plurielles).

Grieveson, Lee, MacCabe, Colin (eds), Empire and film, London, BFI, 2011.

Haynes, Jonathan, "Introduction", in Nigerian video films, ed. by Jonathan Haynes, rev. and exp. ed., Athens, Ohio University Center for International Studies, 1997, p. 1-36.

-, Nollywood: the creation of Nigerian film genres, Chicago, University of Chicago Press, 2016.

Kodjo, François, "Les cinéastes africains face à l'avenir du cinéma en Afrique», Revue tiers-monde, t. XX, 79 (juillet-septembre 1979), p. 605-614.

LARKIN, Brian, "Degraded images, distorted sounds: Nigerian video and the infrastructure of piracy", Public Culture, 16/2 (2004), p. 289-314.

-, Signal and noise: media, infrastructure, and urban culture in Nigeria, Durham/London, Duke University Press, 2008.

Loвato, Ramon, "Nollywood at large", in Shadow economies of cinema: mapping informal film distribution, London, BFI, 2012, p. 55-67. 
Meyer, Birgit, "Ghanaian popular video movies between state film policies and Nollywood", in Viewing African cinema in the twenty-first century: art films and the Nollywood video revolution, ed. by Mahir Şaul, Ralph A. Austen, Athens, Ohio University Press, 2010, p. 42-62.

Miller, Jade L., Nollywood Central, London, Palgrave/BFI, 2016.

Окоме, Onookome, "Nollywood and its critics", in Viewing African cinema in the twenty-first century: art films and the Nollywood video revolution, ed. by Mahir Şaul, Ralph A. Austen, Athens, Ohio University Press, 2010, p. 26-41.

Rouch, Jean, Situation et tendances actuelles du cinéma africain, rapport pour la table-ronde de Venise (10-11 septembre 1961), Paris, UNESCO, 1961.

Şaul, Mahir, Austen, Ralph A. (eds), Viewing African cinema in the twenty-first century: art films and the Nollywood video revolution, Athens, Ohio University Press, 2010.

Simondon, Gilbert, Sur la technique, éd. par Nathalie Simondon, Paris, Presses universitaires de France, 2014.

Turquetr, Benoît, Inventer le cinéma. Épistémologie: problèmes, machines, Lausanne, L'Âge d'Homme, 2014. 